\title{
Negotiating Children's Social Contexts in Jamaica: Ethics, Practicalities and Research Methodologies
}

\section{Joy Moncrieffe}

\begin{abstract}
Conducting research in contexts of violence requires sensitivity and prudence, particularly when the study involves children. This article outlines some of the ethical and practical issues that influenced 'how' researchers worked with children in selected inner-city Jamaican communities. It demonstrates the importance of self-reflection, recognising that researcher's social backgrounds may have much more weight than they acknowledge on the research process and product.
\end{abstract}

\section{Introduction}

This article discusses some of the ethical and practical considerations that influenced methodological choices and approaches in work among children in Jamaica's violent inner-city communities. It demonstrates how researchers' social backgrounds and interests influenced the research process, and emphasises the importance of self-reflection, such that researchers can be aware of the effects of their own socially acquired dispositions. The article highlights some of the personal and professional challenges of working in the heart of violent contexts, particularly with children. It underscores the need for sensitivity to the contexts and to children's vulnerabilities; it also reinforces the importance of respecting children as persons with agency, who can provide critical lessons on the causes and consequences of violence, the spaces for social action and the most productive ways in which external actors can engage.

The first section of this article provides a brief background on Jamaica and introduces the research project; the second sets out and reflects on several implications - for the research process and its outcomes - of working with children in violent contexts. The last section concludes.

\section{Background on Jamaica}

Paradoxically, Jamaica is recognised as a wellestablished democracy and, at the same time, as a country that has a long history of arbitrary and autocratic use of state power. Particularly in the decades after Independence, this was used to instigate political violence (Gray 1991, 2004; Edie 1991), which led to turf wars in the 1970s and 1980s. These violent disputes over turf played out largely within, but then gradually expanded beyond 13 inner-city political strongholds, which Carl Stone (1985) first described as garrisons. Within these areas, gang leaders maintained close relationships with 'responsible' Members of Parliament (MP). On the MPs' behalf, gangs controlled entry and exit and ensured total support for the political party. In return, the community got access to scarce benefits and spoils (Figueroa and Sives 2002; National Committee on Political Tribalism 1997). Although political alignments and connections still exist, the causes of violence are now more diverse. For example, violence related to drugs and extortion has increased substantially (Headley 2002, 2005). With these developments, levels and types of violence have reached unfathomable scales, touching people who were once considered 'off limits': babies and young children, the elderly, members of the clergy and respected professionals.

In 2004, approximately 50 per cent of those incarcerated for major acts of violence were between 15 and 24 years old, while over 80 per cent were between 15 and 35 . Not unlike other 
such contexts, the majority of victims and perpetrators are male, including youth and boys. There are concerns that children within these areas are being socialised in ways that are reproducing violence across generations. Given this background, the original research project, 'Labelling, Violence and Citizenship', was designed to study how differently labelled children are being socialised within these violent contexts. The emphasis on labelling - how people and their perceived roles in society are named - was deliberate. It recognised that different categories of children experience violence in different ways and that children may be assigned certain labels, which influence how they experience violence and citizenship.

However, during the initial focus group discussions, children were instrumental in redefining the core themes of the project. Researchers listened as children stressed that while 'labelling and citizenship' are important for understanding their experiences of violence, it was more significant to study how they experienced and negotiated the physical, social and psychological boundaries of their violent contexts. This did not mean a total redirection of the study since labelling is used to cultivate, express and challenge boundaries. However, the children introduced a more comprehensive and engaging project, which better depicted their realities.

The research project was, therefore, refined to study 'Boundaries and Social Actions'. First, it examined how children who are growing up in a variety of, often, overlapping violent contexts are socialised into different forms of citizenship within the social, psychological and physical boundaries they inhabit. Second, it looked at the conditions under which various groups of children are more and less able to resist their boundaries and even challenge adverse socialisation processes. Importantly, the project focused on children across a range of social contexts, including within middle and upper class areas, which though not untouched by violence are not yet saturated by it. This broad approach was considered important for analysing socialisation processes across different classes including the diverse structural conditions that underpin them. It was also important for studying what is distinct about growing up in contexts where there are relentlessly high levels of violence everyday, in different forms and from multiple sources.

The research underscored some of the ways in which violence is redrawing physical borders and social and cultural boundaries. Children described the multiplicity of boundaries that they experience - physical, social and psychological - and examined how these boundaries are cultivated. The research disaggregated among children, highlighting dimensions of gender, race, class and wealth. It analysed children's actions in the face of boundaries to action (Hayward 2000), emphasising the roles of social conditioning (Bourdieu 1980) in influencing the character of actions and underscoring the point that resistance, although it frequently occurs, may still be shaped by the systems of meanings that children have inherited.

\section{Defining the research strategy}

The core research team comprised a consultant social scientist, who was born and raised in an upper-middle class area; a social worker, who grew up in one garrison community and who, although no longer resident within the area, still locates his business there; and me, born in rural Jamaica, partially educated in the Kingston Metropolitan area but now permanently residing outside of the country and, therefore, largely considered a 'foreigner', both because of my rural heritage and current external residence. Although researchers agreed on many core issues, each offered a distinct interpretive lens, framed by his/her particular social background and subsequent experiences. Each was able to gain access to different constituencies and each had different skills that worked more or less effectively with different groups of children. It proved important, as the research progressed, to apportion tasks in ways that would capitalise on the skills and expertise that each researcher offered and, particularly, to place each researcher in the context where he/she was best able to develop effective rapport with respective groups of children.

As lead researcher, I worked across all social contexts. However, I was - particularly in the earlier stages of the study - much more at ease working with children within the garrison communities, although I did not share the same social background. This comfort came from 
working over very long periods in similar contexts across a range of countries. More importantly, it also came from a personal commitment to understand and respond to perceived need, particularly within the most fragile and ostracised environments. On reflection, I was interested in and understood the languages of need much more than I did the languages of privilege. In contrast, my co-researcher, who originated from an uppermiddle class area, led the research in the more privileged schools and engaged less with children in the garrison areas. The social worker from the garrison community did not work with students from the more privileged communities but was especially instrumental in securing easy access to inner-city schools.

This division in responsibilities proved important to the final research product. We were able to obtain copious amounts of deep and valuable information, by taking maximum advantage of researchers' skills and initial interests. However, in the early stages of the study, it compromised learning within the team itself, for - at least initially - researchers breached their own social boundaries and comfort zones only in very limited ways. This occurred although one core objective of the project was to encourage children to venture beyond their 'boxes' in order to understand and be sensitive to other children's realities. Importantly, as the study progressed and dialogue intensified within the team, researchers felt personally challenged to develop a deeper understanding of the social worlds they were unaccustomed to. This commitment led to increased collaboration and less emphasis on distinct spheres of influence.

However, it did not prevent conflicts. For example, the consultant social researcher (from the upper-middle class area) and the social worker (who originated from one garrison area) disagreed on key aspects of the research strategy with such intensity that the social worker eventually abandoned the study. In his bid to promote social learning across groups of children, the social worker proposed that our research strategy should include immersions, which would involve transporting upper class children to 'garrison' schools and encouraging dialogue among the children. The consultant vehemently disagreed, arguing that she could not accept such a strategy, as she would not allow her own children to visit a garrison area; the social worker interpreted this as snobbery. Eventually, researchers agreed to host 'conversations' among groups of children across social classes but to do so in a 'neutral' area.

Thus, the forms and levels of collaboration and contestations within the research team itself demonstrate the folly of downplaying the weight of social conditioning and 'positionality', not only on the researched but also on the researcher.

\section{Children as subjects and actors}

Critically, members of the team were all committed to ensuring that the research project involved children and the elderly as both subjects and actors. The research methodologies we devised combined quantitative and qualitative tools, comprising focus group discussions, unstructured conversations and selected life stories with 6-9, 10-13 and 14-17-year-olds from two garrison communities. They also included personal interviews, a series of unstructured conversations with community members and teachers and modest use of participatory video. The community interviews included parents and grandparents. Some grandparents were then selected for in-depth life stories, in order to obtain their perspectives on the causes for the apparent 'transmission of violence' across some families. Interviews were also conducted with government and donor agency representatives who are involved in violence reduction programmes. Here, the aim was to analyse how various agencies intervene in these communities and the extent to which they are able to cultivate spaces for change. As actors, children were to have a crucial role in conducting the research and in formulating and implementing appropriate policy responses.

Quantitative surveys were designed to provide information on incidence of violence, levels of violence, education levels, livelihood choices and violence, gender and violence, age and violence, perceptions and experiences of violence and experiences. Older children were selected and trained to conduct the surveys. Researchers anticipated that this would result in a form of capacity building as individuals and potentially contribute to wider transformational effects. Furthermore, the research project was designed to facilitate children's direct engagement with policymakers and key civil society organisations. All these components were important to the 
team's objective of actively using the research process to help with building the relationships that are necessary for transformational change.

This firm commitment to prioritise children's voices and to include them substantively in the research shaped both the process and the final product in important ways. For example, the scheduled order of the quantitative and qualitative work was inverted, so that, via the rapport built through initial qualitative work, children could be involved in developing key questions to cover in the quantitative survey. As another example, the Violence, Participation and Citizenship (VPG) ${ }^{1}$ group had envisioned that researchers would work within the 'communities', using research to introduce participatory processes, which could then trigger positive social actions. However, when they were consulted in the initial stages of the research, children emphasised the difficulties of negotiating the physical boundaries within their 'imagined communities'. They stressed that they felt 'safer' within their schools than in the spaces they reside. Within their 'communities', children were often segregated depending on popular perceptions of their parents' or guardians" political allegiances or any other conceived reasons for turf divisions. Such was the intensity of the divisions that children who lived in one lane could be prevented from consorting with others who lived merely three blocks away. Those who dared to traverse the boundaries could be killed. Schools, in contrast, included children across factions in a comparatively safe space.

All the interviews within middle and upper class communities were also conducted within schools, for various different reasons. Social dynamics in many upper class areas are different from those in the inner-cities. In inner-city areas, residents are accustomed to and enjoy communal style living during periods of peace. Characteristically, upper class areas are more protective of their private spaces. Violence exacerbates this: many people live in intense fear of violent crimes and have retreated to gated communities farther into the hills. Consequently, the schools provided the easiest access to middle/upper class children.

\section{Selecting the children: the weight of social boundaries}

From the earliest stages of the study, researchers recognised and were forced to negotiate differing social boundaries. Jamaica is an intensely socially divided society and this was demonstrated, quite clearly, in the distinct procedures required to gain access to 'inner-city' and middle/upper class students. In inner-city schools, it was sufficient to contact the schools' principals and to supply a letter stating the purpose of the research. For all inner-city schools, consent was given without parental permission. Once principals agreed, teachers marched into classrooms and told students to participate in our discussions. In upper class areas, by contrast, researchers were required to write very detailed letters, including all the research questions. Potentially sensitive questions had to be removed, if instructed. The finalised letters and questions were then sent to the parents, who gave or withheld permission. Only children who returned permission slips were allowed to attend. Furthermore, unlike inner-city areas, a teacher was required to monitor the interviews and conversations. Although the research team benefited from this 'ease of access' to children within the innercities, the situation presented a moral dilemma since we recognised that we were, in fact, capitalising on these children's lesser experiences of rights and respect (compared with children in the middle to upper classes), while one of the project's core objectives was to expose and bridge the boundaries that caused certain categories of children to experience rights and citizenship in such very distinct ways. Therefore, researchers felt obliged to ensure that this 'unfair advantage' was used to benefit the children within the inner-cities. This was principally achieved through carefully targeted publications which used children's voices to depict their experiences of violence, and supportive actions such as employing a psychologist to work with traumatised children.

\section{Understanding boundaries: speaking the languages of privilege and need}

Having gained access to the children, researchers then had the challenge of understanding the deep cultures and socialisation processes within and across communities. The schools in middle/upper and inner-city areas presented immediate visible contrasts, such as of wealth and neglect. The cultures within the schools appeared to reflect the cultures within communities. As the children confirmed, residents within middle and upper class communities tend to maintain more 
private, anonymous lives as opposed to children within garrison areas, who are often raised in more communal, African-style settings, with all the camaraderie and conflicts that this nearness breeds. As a consequence, many children within the garrison areas could tell each other's stories, were familiar with the same events and were, therefore, able to challenge and/or confirm the veracity of certain positions. In contrast, while there were clear friendships within upper and middle class schools, children appeared to maintain fairly distinct lives and to have been socialised to refrain from divulging too many private domestic issues. Such differences in cultures - openness versus privacy, and close connections versus distance - substantially affected the study and required that researchers employ very different techniques.

Much time was spent throughout the study on refining the questions being asked and the approaches to asking those questions. Violence is a sensitive subject in any context but special care is required when the focus is on children.

Domestic violence was a particularly difficult subject to broach and, in middle and upper class schools, head teachers advised that parents would not allow their children to respond to questions on this subject. As in other studies of violence (Moser and McIlwaine 2004), children were encouraged to use drawings and drama to depict their feelings. Predictably, children in middle and upper class schools were reluctant to discuss the issue. Research among middle and upper class children presented other challenges for, in these groups, children were normally more guarded and it required skill and sensitivity to learn about these children's fears of violence, the social boundaries they experienced, particularly in gated communities and their own experiences of violence, though this was not as relentless as it was in the inner-cities. Eventually, researchers learnt about the needs that existed within contexts of privilege and discovered some largely unexposed commonalities among children who lived in garrison and more privileged areas.

In contrast, researchers were unprepared for the raw candour, including open expressions of trauma, from children in garrison areas - some as young as five years old - who have close and intimate knowledge of violence, death and loss. Almost every child had lost a family member; some had lost many.
Girl (six years):

My mother and my father died (sobbing). My mother dead by gun shots.

When did it happen?

From I was 2 years old, Miss, and me [I] don't know her.

How did your father die?

Don't know; they don't tell me about him.

Boy (eight years):

Miss, my 2 brothers died. One went off with my father and someone shoot him off in the water and him drown [someone shot him and he drowned]. The other one, it's the police who shoot him.

Why did it happen?

I don't know why it happened. The last time, I was sleeping and my mother woke me and told me that my brother died. He was 16.

(Moncrieffe 2007: 23)

Each child gave vivid descriptions of the multiple and overlapping boundaries they encountered each day. Researchers recognised that as sociologists and political scientists they were not equipped to deal with the real needs the children expressed. Consequently, the team employed a psychologist, who could provide services for children who needed and desired it.

\section{Negotiating boundaries; promoting social action}

Like other teams within the VPC, this research

project was not merely concerned with amassing data. The study had the purpose of instigating actions among researchers themselves, children and policy actors. It became increasingly clear throughout the course of the project that constructive social action, of the sort that the VPC envisioned, depended upon understanding the substantive boundaries to action within and across communities, including the ingrained norms, perceptions and labelling of 'others' that block recognition of boundaries and effective responses to them. Having recognised the boundaries to action within the research team itself, as well as the process of change required for transformation, the team developed more deliberate strategies to encourage social action. The focus of interventions changed from immediate communication with policymakers to addressing the learnt psychologies of fear and the effects of the personal traumas that children 
encountered. Psychological interventions in schools have been proving to be critical here.

Furthermore, it was urgent to begin a process of addressing social boundaries. Consequently, researchers hosted a public discussion among all schools in the Kingston and St Andrew area. The session allowed for exchanges among children from different social classes and allowed different groups to confront, challenge and even begin to change some of the strident and false perceptions they held of 'others'. In addition, rather than immersions, researchers opted for a radio programme, in which children from various social backgrounds could converse anonymously, though via public airwaves, thus affording them the opportunity to express themselves from a 'safe space'.

\section{Conclusion}

Research methodologies work most effectively when they are responsive to their contexts.

Responsiveness to the contexts and to the people within them might require challenging researcher's personal frameworks; personal transformations may occur, as investigators learn to breach their own socially acquired boundaries. Furthermore, genuine dialogue in social research might require that people are involved, not only as subjects but as actors. Where they are respected as actors, the researched are able to offer unanticipated perspectives and to participate in unanticipated ways. This level of participation may not always lead to desired or desirable outcomes but, with skill, can be honed to achieve productive results. For example, with the children's lead, the research developed into an interesting analysis of 'boundaries and social actions', which includes the construction of selfhood in contexts of violence. This new theme encapsulates labelling but is more wide ranging. The project was renamed, since it became clear that boundaries - physical, social, economic and psychological - were the most significant barriers to citizenship and, correspondingly, the cause of distinctly different citizenship experiences across social classes in Jamaica. Negative labelling across classes contributed to these boundaries but was also a product of these boundaries, particularly among children. Furthermore, these psychological boundaries impeded social action and had to be addressed if there were to be substantive changes arising from this project or from other stimuli.

As the article describes, the methodologies used in the project aimed to do more than gather data; they were key to a larger action-oriented agenda, which sought to contribute to already existing processes for change. Children were included, not merely as subjects but as actors, including researchers. While the children's roles in conducting the quantitative survey may have sacrificed some rigour (Kemmis and McTaggart 2000), children were, demonstrably, less inhibited speaking with their peers and many expressed appreciation for the opportunities to give meaningful input. The added value of including children in such a substantial way cannot be quantified. Similarly, researchers were concerned to avoid amassing information without responding to the palpable needs among children who have been traumatised by violence. Psychological interventions were necessary, not only to deal with trauma but to children's selfactualisation as citizens. We therefore considered it ethical to include the child psychologist in the team, not merely to assist children who were traumatised but to provide continual service to the schools after the project ended. In addition, information sharing and cross-group dialogue was considered essential for challenging stereotypes. Other methodological choices were made as the research progressed and it became clear how the project could contribute to actions.

Research methodologies are not set formulae; they can and should be adapted, particularly when they are part of a social action strategy. Only with knowledge of the contexts and the people within them can researchers make sensible decisions about the types of methodological choices and approaches that are best able to instigate constructive social actions or impede them. 


\section{Notes}

1 For more details on the Violence Citizenship and Participation group's research, see McGee, this IDS Bulletin.

2 Children may live with guardians rather than parents for a variety of reasons. It is common throughout the Caribbean for parents to negotiate with better-off family members to

\section{References}

Bourdieu, P. (1980) The Logic of Practice, Stanford, California: Stanford University Press

Edie, C.J. (1991) Democracy by Default: Dependency and Clientelism in Jamaica, Kingston: Ian Randle Publishers

Figueroa, M. and Sives, A. (2002) 'Homogenous Voting, Electoral Manipulation and the "Garrison" Process in Post-Independence Jamaica', Commonwealth and Comparative Politics 40.1: 81-8

Gray, O. (2004) Demeaned but Empowered: The Social Power of the Urban Poor in Jamaica, Kingston: University of the West Indies Press

Gray, O. (1991) Radicalism and Social Change in Jamaica 1960-72, Tennessee: University of Tennessee Press

Hayward, G.R. (2000) De-Facing Power, Cambridge: Cambridge University Press

Headley, B. (2005) Deported, Volume 1, Kingston: Stephenson's Litho Press care for children whom they think would not fare as well in their own care. However, particularly in the inner-cities in Jamaica, children may be forced to live with members of their extended families or family friends where - principally - their fathers are killed or imprisoned or where parents have migrated in search of work.

Headley, B. (2002) Essays on Crime and the Politics of Jamaica, Kingston: LMH Publishing Limited

Kemmis, S. and McTaggart, R. (2000)

'Participatory Action Research', in N. Denzin and Y. Lincoln (eds), Handbook of Qualitative Research, London: Sage

Moncrieffe, J. (2007) Making and Unmaking the Young Shotta [Shooter]: Boundaries and (Counter)Actions in the 'Garrisons', IDS Working Paper 297, Brighton: IDS

Moser, C. and McIlwaine, C. (2004) Encounters with Violence in Latin America: Urban Poor Perceptions from Colombia and Guatemala, London: Routledge:

National Committee on Political Tribalism (1997) Report of the National Committee on Political Tribalism (Under the Chairmanship of Justice James Kerr), July, Kingston, Jamaica

Stone, C. (1985) Democracy and Clientelism in Jamaica, New Jersey: Transaction Books 